\title{
Design of Shift Register Using Organic Thin Film Transistor for Gate Driver of Flexible Organic Light-Emitting Diode Displays
}

\author{
Seung-Il Cho and Makoto Mizukami* \\ Innovation Center for Organic Electronics, Yamagata University, \\ 1-808-48, Arcadia, Yonezawa, Yamagata 992-0119, Japan \\ cho_si@yz.yamagata-u.ac.jp,m_mizukami@yz.yamagata-u.ac.jp
}

\begin{abstract}
Interest in flexible organic light-emitting diode (OLED) displays is increasing. Fabrication methods based low-temperature, low-cost, and large-area printing processes have been studied to simplify the manufacturing process. A gate driver is required to drive the OLED display. The gate driver is integrated into the panel to reduce the manufacturing cost of the display panel and to simplify the module structure.

In this study, a shift register is proposed using organic thin-film transistors (OTFTs) to integrate flexible an OLED display with the gate driver. OTFT modeling was performed using the experimental data with a three-terminal silicon-on-sapphire field effect transistor (SOSFET) model. Furthermore, a shift register for the gate driver was designed using the required process design kit with the OTFT modeling data. Operation of the shift register was confirmed by HSPICE simulation. The layout of the shift register was designed using the solution process rule for OTFTs. Those results show the possibility of gate diver integrated in flexible OLED display.
\end{abstract}

Keywords: gate driver, organic thin film transistor, pseudo logic, silicon-on-sapphire field effect transistor model, circuit simulation, flexible organic light emitting diode display

\section{Introduction}

Living labs have recently received significant attention and are being increasingly adopted for urban/suburban lifestyle innovations using wearable devices, ubiquitous devices, information and communication technology (ICT), and internet of things (IoT) service [1-2]. Furthermore, there have been innovative studies on environmentally friendly organic electronics and printing technologies [3-5]. Among these technologies, organic light-emitting diodes (OLEDs), organic thin-film transistors (OTFTs), and organic photovoltaics (OPVs), which use organic materials, have been investigated for flexible devices [6-8], and their development is underway. In particular, interest in flexible OLED displays is increasing [9-11]. An institution of Yamagata University, Smart MIRAI House, was built to demonstrate the research results for living labs and the living environment of the near future, as shown in Figure 1 [12]. A large-scale wall display of the institution will be developed for video conferences, video telephony, and other communication media using the organic material printing technology.

Fabrication methods based on low-temperature, low-cost, and large-area printing techniques that utilize inkjets, screens, gravures, and reverse-offsets have been studied to simplify the manufacturing process as compared to conventional vacuum or photolithography processes [13-16]. However, production techniques for organic and printed electronics are not yet standardized. A required process design kit (PDK) for

Received (December 15, 2017), Review Result (February 2, 2018), Accepted (February 6, 2018)

${ }^{*}$ Corresponding Author 
OTFTs does not exist for integrated circuit design and simulation. Therefore, a PDK in flux is needed for circuit simulation.

A gate driver is required to drive the OLED display. In order to reduce the manufacturing cost of the display panel and to simplify the module structure, a gate driver is integrated into the panel [17-19]. Thus, an integrated circuit (IC) bonding technology on the flexible panel is required. The problem associated with packing for the flexible panel should also be solved. Therefore, it is necessary to fabricate an integrated gate driver using an OTFT that can be manufactured by a panel process.
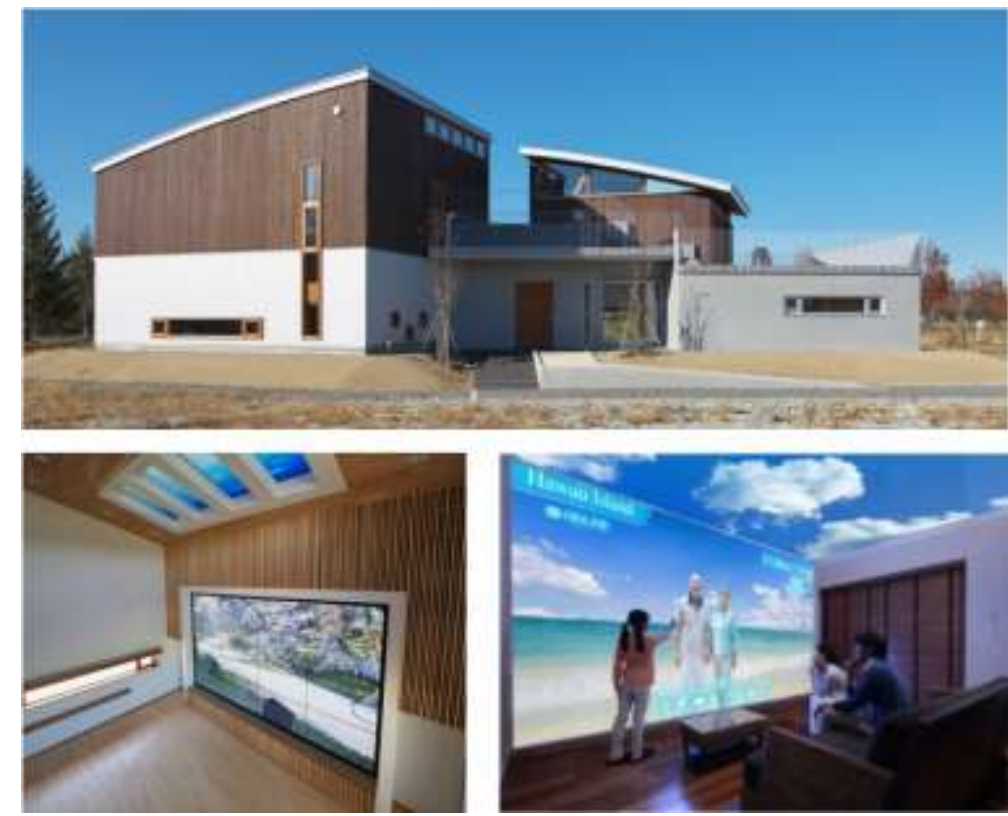

Figure 1. Large-Scale Wall Display of Smart MIRAI House

In this paper, the gate driver using OTFTs is proposed to be integrated with the flexible OLED display. OTFT modeling was performed by measuring transfer characteristics of OTFTs. A shift register for the gate driver was designed using the experimental data. The operation of the shift register was confirmed by circuit simulation. Moreover, the layout design of the shift register was realized using the solution process rule for OTFTs.

The remainder of this paper is organized as follows. Section 2 describes the implementation of prototype flexible OLED displays with an OTFT backplane. OTFTs were manufactured for evaluation of the transfer characteristics. Moreover, the manufacture and measurements of a pseudo complementary metal oxide semiconductor (CMOS) inverter gate are discussed in Section 3. Section 4 reports the simulation results for the designed shift register by HSPICE with OTFT modeling PDK. The layout design of the shift register is also achieved. Finally, Section 5 concludes the paper.

\section{Flexible OLED Displays with an OTFT Backplane}

We have succeeded in the development of a solution-processed OTFT backplane for a flexible multiphoton emission OLED display, as shown in Figure 2. In 2015, a 1.8-inch grayscale flexible multiphoton emission OLED display (Figure 2 a) with an active-matrix OTFT backplane was developed using solution-shearing and solution processes [9-10]. Careful lyophobic/lyophilic processing of the insulating material, bank structure, and organic semiconductor (OSC) ensured high performance of the OLED display. The flexible OLED display and drive circuits were connected via a flexible printed circuit. Both images and videos were successfully displayed, even under bending. 


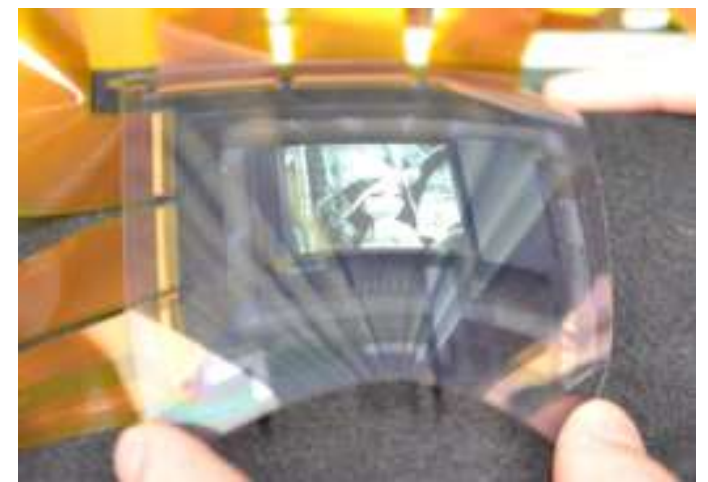

(a) 1.8-Inch Grayscale

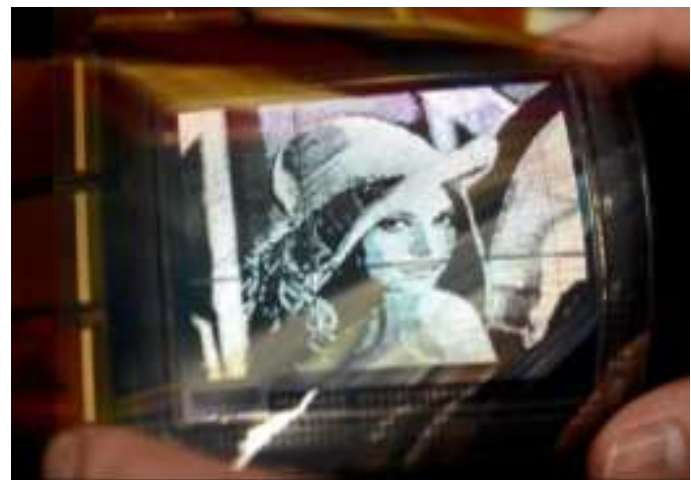

(b) 3.2-Inch Grayscale

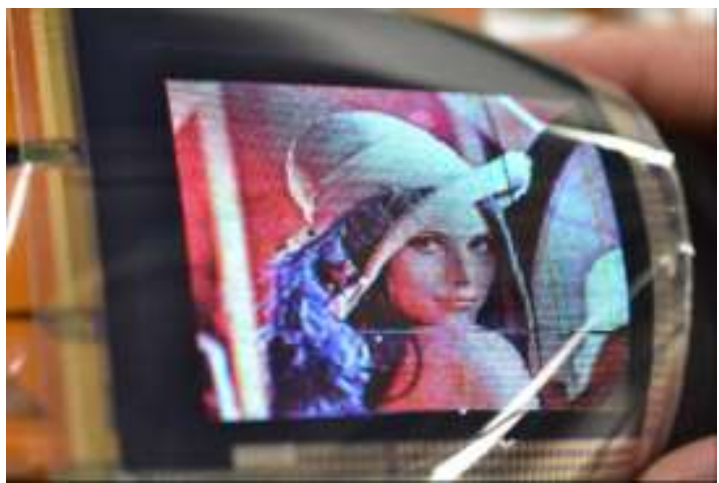

(c) 3.2-Inch Color

Figure 2. Prototype of Flexible OLED Displays with the OTFT Backplane

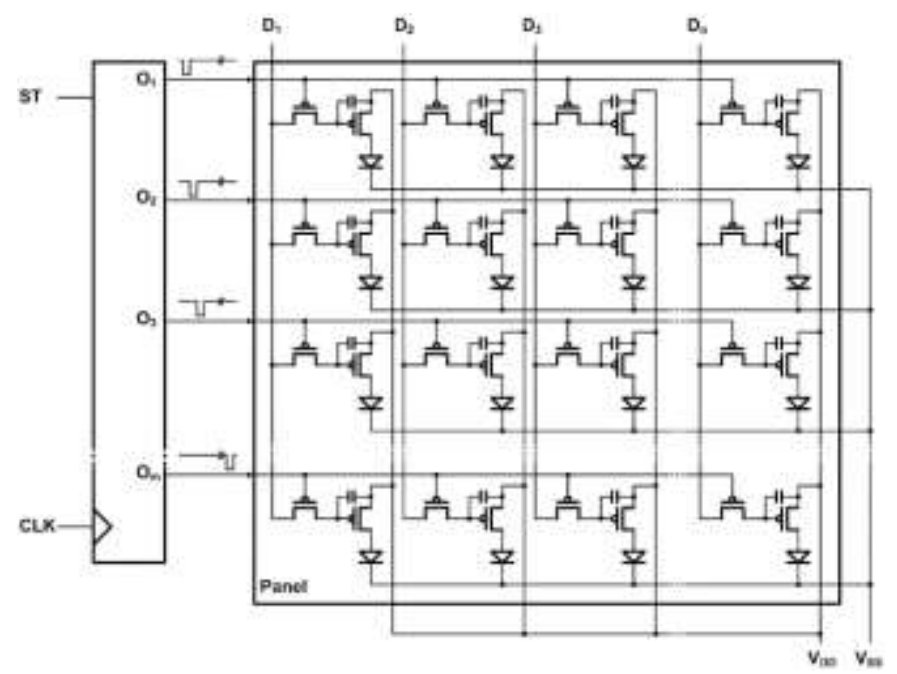

Figure 3. Block Diagram of the Gate Driver Integrated in the OLED Display

After a year, in 2016, we increased the size of the OLED display (Figure 2 b) with the OTFT backplane to 3.2-inch using inkjet printing technology and solution processes. Recently, in 2017, our team succeeded in the development of a 3.2-inch flexible color OLED display (Figure $2 \mathrm{c}$ ) with an OTFT backplane using inkjet printing technology and solution processes [10-11]. OSCs were applied using the inkjet printing technique. The mobility of the OTFTs formed by coating the OSC solution using inkjet printing was about three times larger than that obtained using the solution-shearing method. Both images and videos were successfully displayed, even under bending. In order to reduce 
the manufacturing cost of the display panel and to simplify the module structure for flexibility, the gate driver was integrated into the panel. A gate driver (Figure 3) using ptype OTFTs was needed to drive the OLED display and to be integrated on the plastic substrate.

\section{Pseudo-CMOS Logic using OTFT}

\subsection{Structure and Transfer Characteristics of OTFT}

Figure 4 shows the structure of an OTFT that has a bottom-gate/bottom-contact (BGBC) configuration. A 50-nm-thick Al gate electrode layer was prepared by vacuum deposition with a metal mask on a glass substrate. For the gate dielectric layer, a 400-nmthick film of cardo-polymer (GIP-2001/P2.5K; Nippon Steel \& Sumikin Chemical Co., Ltd.) was formed by a spin-coating solution process [20]. Au source/drain (S/D) electrodes with a thickness of $50 \mathrm{~nm}$ were prepared by vacuum deposition. The electrodes were patterned using conventional photolithography and wet etching. The OSC layer (Lisicon S1200; Merck Ltd.) was formed by spin-coating. For protection, the Cytop (Asahi Glass Co.) layer was subsequently spin-coated onto the OSC layer. The OSC and protection layer were patterned using photolithography and dry etching.
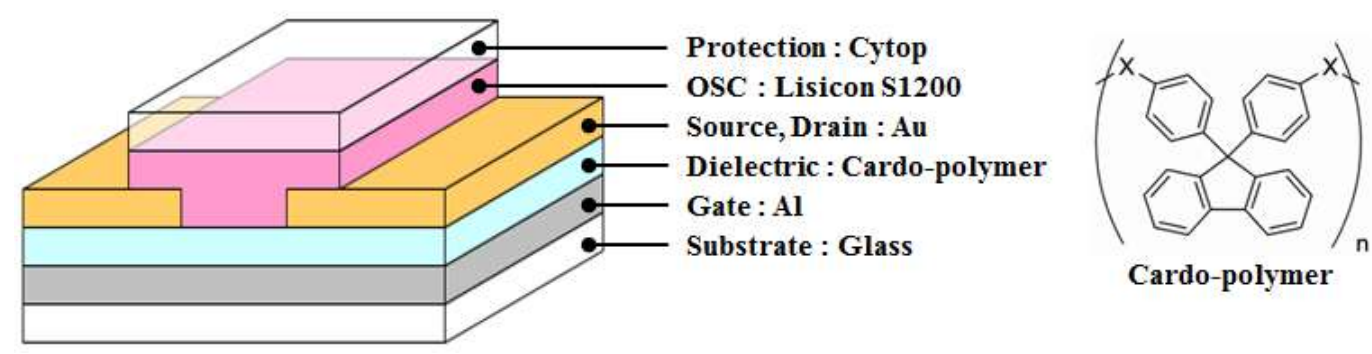

Figure 4. Schematic of OTFT Structure

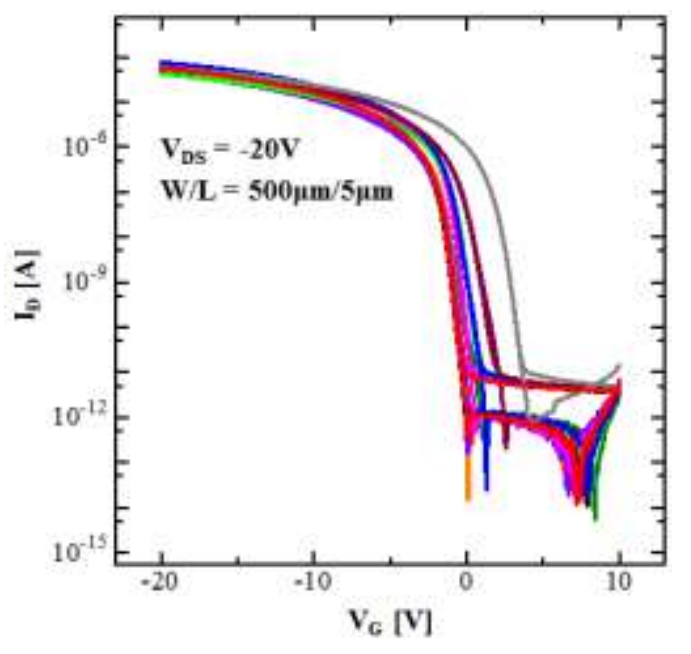

Figure 5. Transfer Characteristics of OTFT

Figure 5 shows the electrical transfer characteristics of 12 depletion-type OTFTs measured in saturation at a drain-to-source voltage of $-20 \mathrm{~V}$. The field-effect mobility, on/off current ratio, and threshold voltage $\left(\mathrm{V}_{\text {th }}\right)$ were $0.42 \mathrm{~cm}^{2} /(\mathrm{V} \cdot \mathrm{s}), 3.4 \times 10^{7}$, and $-0.2 \mathrm{~V}$, respectively. 


\subsection{Pseudo-CMOS Logic; Inverter}

The pseudo-CMOS logic is a logic gate that uses monotype OTFTs [7, 21]. The pseudo-inverter circuit produced herein consisted of four p-type OTFT devices with different channel widths (W), as shown in Figure 6 (a). The $\mathrm{W}$ values of $\mathrm{M}_{1}$ and $\mathrm{M}_{2}$ were 500 and 1,500 $\mu \mathrm{m}$, respectively. The $\mathrm{W}$ values of $\mathrm{M}_{2}, \mathrm{M}_{\mathrm{UP}}$, and $\mathrm{M}_{\mathrm{DP}}$ were the same. Figure 6 (b) shows a photograph of the fabricated device. Figure 6 (c) presents an experimental result. $\mathrm{V}_{\mathrm{DD}}, \mathrm{V}_{\mathrm{SS}}$, and $\mathrm{V}_{\text {in }}$ were $15,-15\left(-\mathrm{V}_{\mathrm{DD}}\right)$, and $0 \sim 15 \mathrm{~V}$, respectively, for a $10-\mathrm{Hz}$ square wave. Operation of the inverter with a full-swing between $\mathrm{V}_{\mathrm{DD}}$ and GND was confirmed.

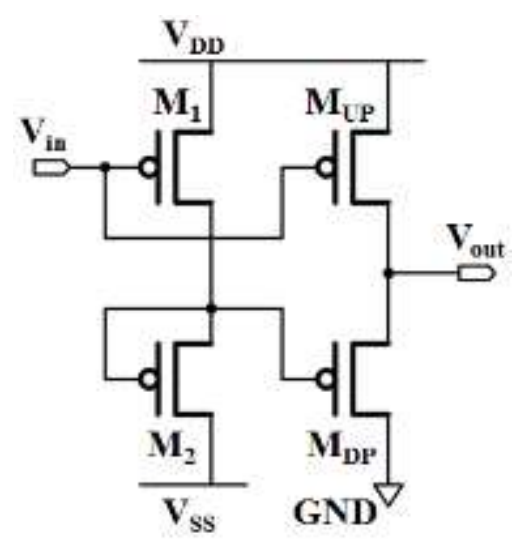

(a) Circuit Schematic

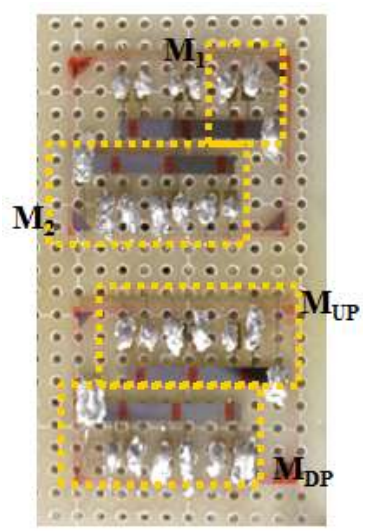

(b) Photograph of the Device

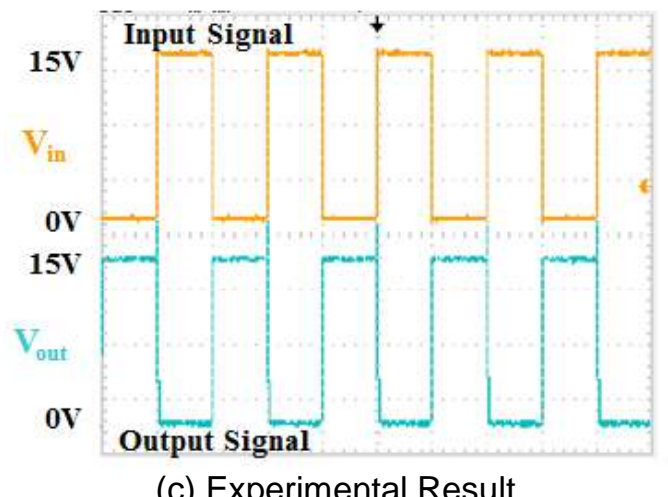

(c) Experimental Result

Figure 6. Pseudo-CMOS Inverter

\section{Design of the Gate Driver using OTFT}

\subsection{OTFT Modeling}

A dedicated PDK is required for circuit design and computer simulation because the structure of an OTFT is different from that of a conventional silicon CMOS. Therefore, OTFT modeling is performed by using the measured transfer characteristics of the OTFTs. Thus, a PDK is produced for circuit design and computer simulation. 
Table 1. Parameters of PDK for OTFT Circuit Simulation

\begin{tabular}{|l|c|c|c|}
\hline Parameter & Fast mode & Typical mode & Slow mode \\
\hline Library (Depletion type) & PDF & PDT & PDS \\
\hline Vth (VTO) & -2.0 & -0.2 & -0.1 \\
\hline Mobility (UO) & 4.2 & 0.42 & 0.21 \\
\hline Dielectric thickness (TOX) & $4.1 \mathrm{e}-7$ & $4.1 \mathrm{e}-7$ & $4.1 \mathrm{e}-7$ \\
\hline
\end{tabular}

A conventional silicon CMOS includes four terminals because it applies a bias voltage to the substrate of the wafer to prevent leakage current, as shown in Figure 7 (b). Since the OTFT was fabricated on a glass or plastic substrate, a three-terminal silicon-onsapphire field effect transistor (SOSFET) model (MOSFET: level 27 SOSFET) that did not apply a bias voltage to the substrate was used, as shown in Figure 7 (c) [22-23]. Moreover, a PDK was created by applying the parameters derived from the measured values for the OTFT. A PDK in flux can be used to change the parameters depending on OTFT materials. Table 1 shows the parameter values applied in this study.

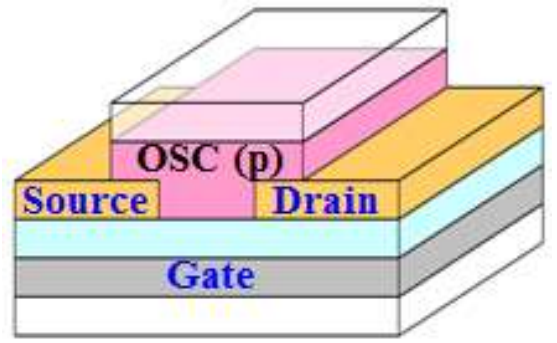

(a) Three-terminal OTFT

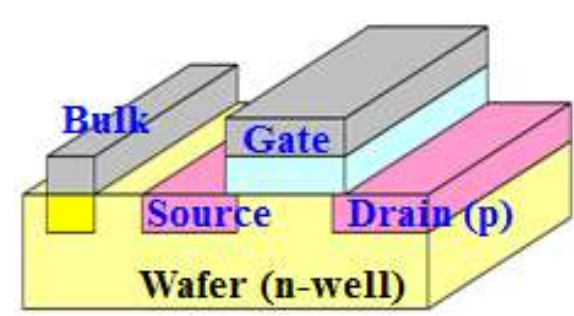

(b) Four-terminal MOSFET

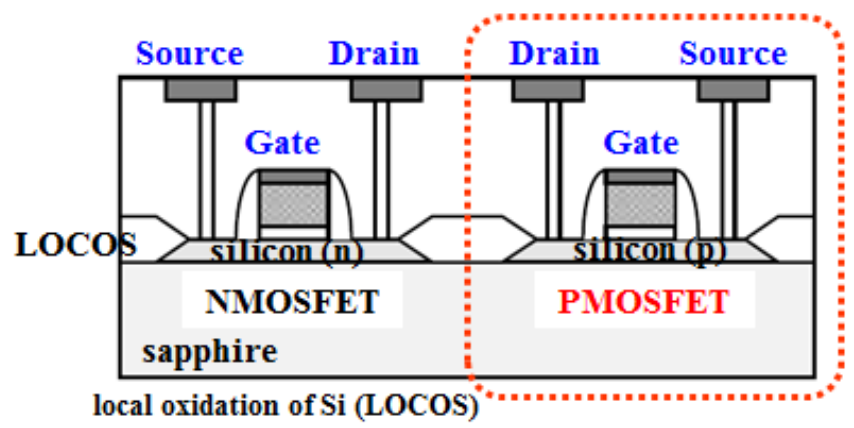

(c) Three-terminal SOSFET Model

Figure 7. Structure of Transistor Models

\subsection{Simulation Results of the 4-bit Shift Register for the Gate Driver}

Figure 8 shows the gate driver for the active-matrix OTFT backplane of the flexible OLED display. The shift registers consisted of a positive-edge D-flipflop with a pseudoCMOS inverter and 7 NANDs. $\mathrm{V}_{\mathrm{DD}}, \mathrm{V}_{\mathrm{SS}}$, and CLK were $15,-15,0 \sim 15 \mathrm{~V}$, respectively, at a $10-\mathrm{kHz}$ square wave. Operation of the shift register was confirmed through the HSPICE simulation, as shown in Figure 9. The output signals of the gate driver should be a sequentially shifted low signal so that the switching OTFT of the backplane is 
sequentially turned on, because the backplane of the flexible display comprises p-type OTFTs.

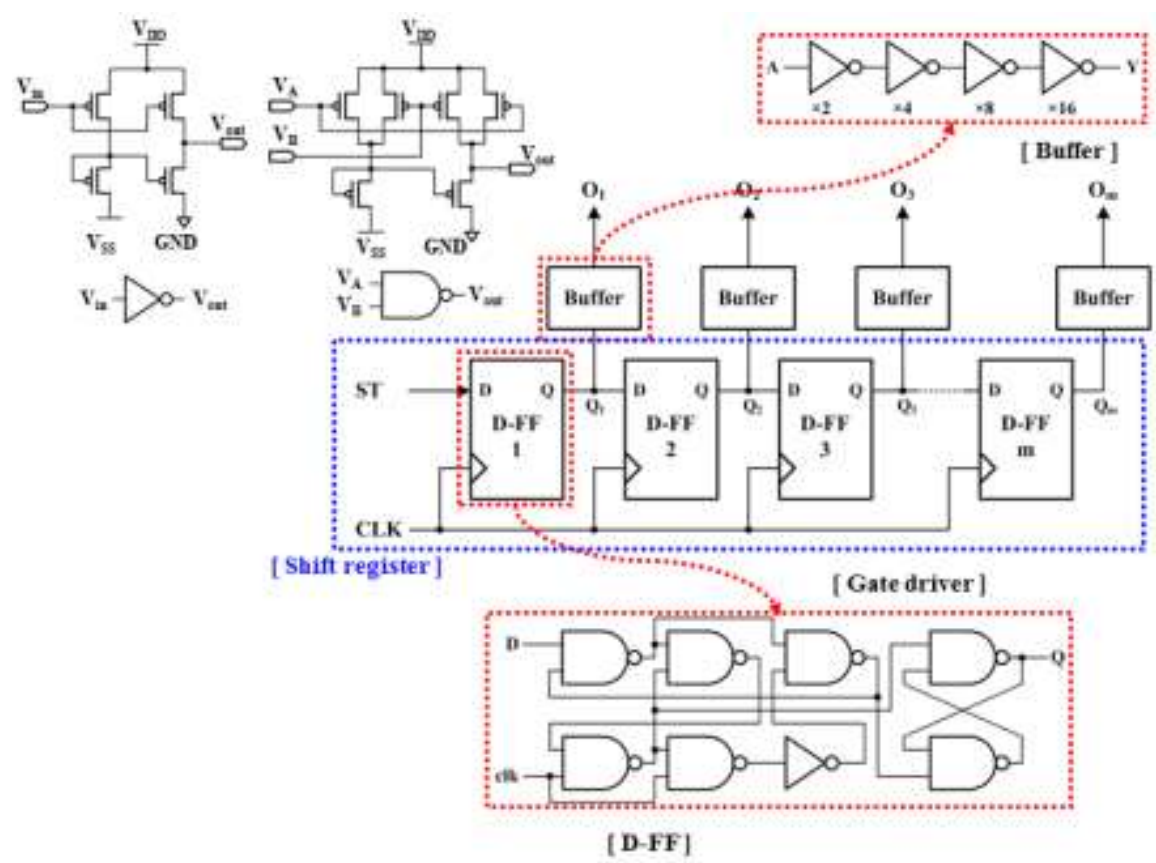

Figure 8. Gate Driver using Pseudo Logic for OTFT Backplane of Flexible OLED Display
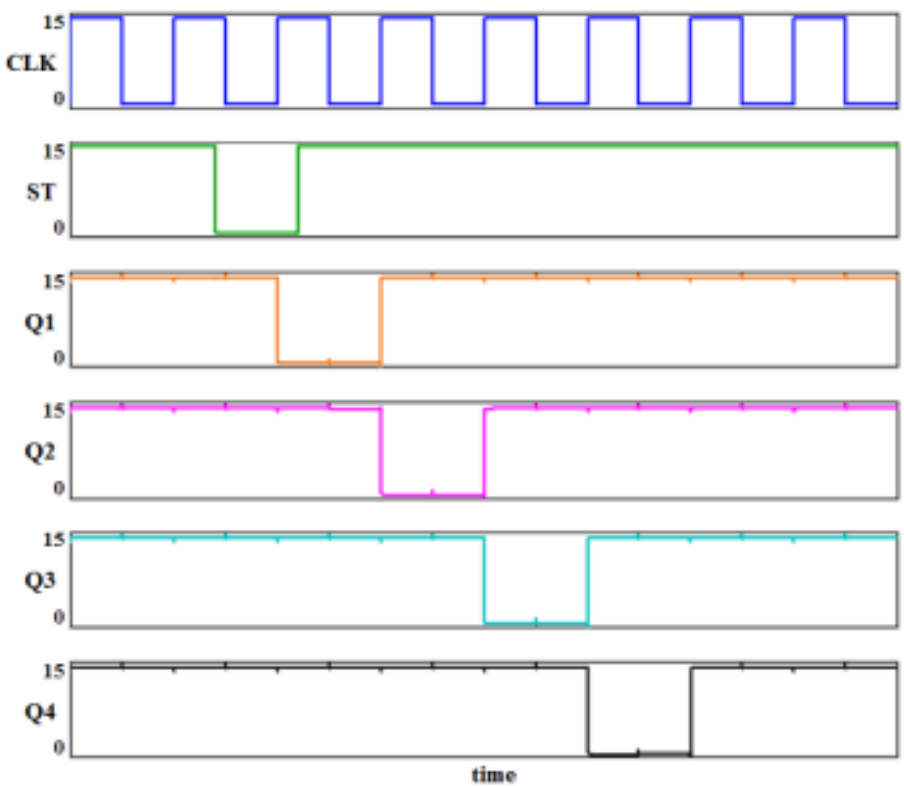

Figure 9. Simulation Results of the 4-bit Shift Register

\subsection{Layout of the Shift Register for the Gate Driver}

Figure 10 shows the layout design of the 4-bit and 32-bit shift registers based on the solution process rule for OTFT. A layout consisted of 5 layers, namely, the gate, dielectric (including the contact hole layer), S/D, Bank, and OSC layers. The layout versus schematic (LVS) was verified. Shift registers will be fabricated using the layout and measured in future research. 

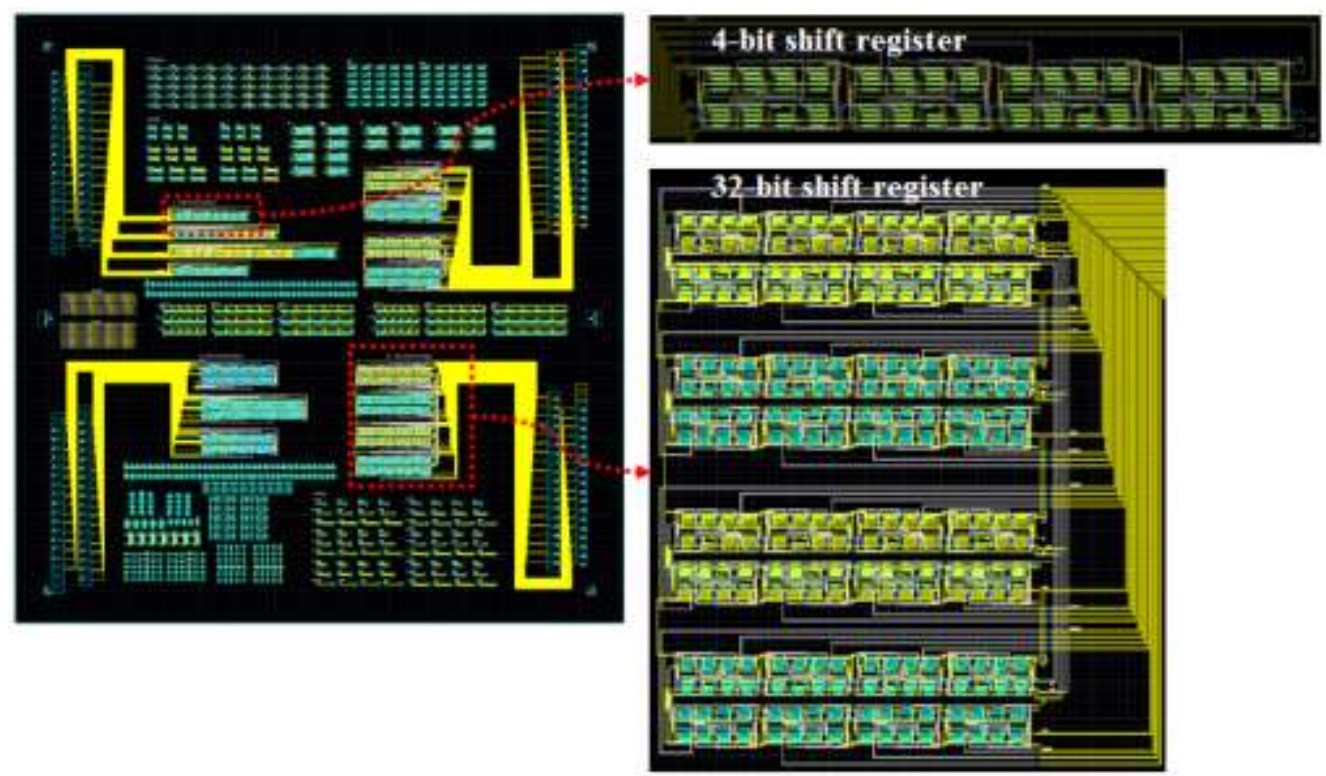

Figure 10. Layout of Shift Registers

\section{Conclusion}

For the flexible OLED display with the active-matrix OTFT backplane, it is necessary to fabricate an integrated gate driver using monotype OTFTs that can be manufactured by a panel process. In this study, the gate driver that integrated into the flexible OLED display is proposed using pseudo-CMOS gates. OTFT modeling was performed using the experimental data. Furthermore, a shift register for the gate driver was designed using a PDK with the OTFT modeling data. Operation of the shift register was confirmed by the HSPICE simulation. Moreover, the layout design of the shift registers was realized using the solution process rule for OTFT. The results confirmed the operation of the gate driver integrated into the flexible OLED display. In future work, we will fabricate shift registers using the layout and measure them. Moreover, we plan to integrate the flexible OLED display with the gate driver.

\section{Acknowledgments}

This paper is a revised and expanded version of a paper entitled "Design of Gate Driver using Organic Thin Film Transistor for Flexible OLED Displays" presented at the 21st International Conference on Electronic \& Information Communication, Goesan, South Korea, November 10-11, 2017.

The VLSI chip in this study has been fabricated in the chip fabrication program of VLSI Design and Education Center (VDEC), the University of Tokyo in collaboration with Rohm Corporation and Toppan Printing Corporation.

The authors would like to thank Miho Abiko, Kaori Watanabe, and Kaori Ito for the process development and implement of OTFT backplane for flexible OLED display.

\section{References}

[1] M. Alba, M. Avalos, C. Guzmán and V. M. Larios, "Synergy between Smart Cities' Hackathons and Living Labs as a Vehicle for Accelerating Tangible Innovations on Cities", Proceedings of 2016 IEEE International Smart Cities Conference (ISC2), 16355382, (2016).

[2] S. Leminen, A.-G. Nyström, M. Westerlund and M. J. Kortelainen, "The Effect of Network Structure on Radical Innovation in Living Labs", Journal of Business \& Industrial Marketing, vol.31 no.6, (2016), pp.743-757.

[3] [3] C.D. Dimitrakopoulos and P.R.L. Malenfant, "Organic Thin Film Transistors for Large Area Electronics”, Advanced Materials, vol.14, no.2, (2002), pp.99-117. 
[4] T. Someya, Y. Kato, S. Iba, Y. Noguchi, T. Sekitani, H. Kawaguchi and T. Sakurai, "Integration of Organic FETs with Organic Photodiodes for a Large Area, Flexible, and Lightweight Sheet Image Scanners", IEEE Transactions on Electron Devices, vol.52, no.11, (2005), pp.2502-2511.

[5] E. Cantatore, T. C. T. Geuns, G. H. Gelinck, E. V. Veenendaal, A. F. A. Gruijthuijsen, L. Schrijnemakers, S. Drews and D. M. de Leeuw, "A 13.56-MHz RFID System Based on Organic Transponders", IEEE Journal of Solid-State Circuits, vol.42, no.1, (2007), pp.84-92.

[6] T. Chiba, Y.-J. Pu and J. Kido, "Organic Light-Emitting Devices with Tandem Structure", Topics in Current Chemistry, vol. 374, no. 3, (2016), pp.1-17.

[7] T.-C. Huang, K. Fukuda, C.-M. Lo, Y.-H. Yeh, T. Sekitani, T. Someya and K.-T. Cheng, "PseudoCMOS: A Design Style for Low-Cost and Robust Flexible Electronics", IEEE Transactions on Electron Devices, vol. 58, no.1, (2011), pp.141-150.

[8] D. Yang, H. Sasabe, Y. Jiao, T. Zhuang, Y. Huang, X. Pu, T. Sano, Z. Lu and J. Kido, "An Effective $\pi-$ Extended Squaraines for Solution Processed Organic Solar Cells with High Efficiency”, Journal of Materials Chemistry A, vol.48, (2016), pp.18931-18941.

[9] M. Mizukami, S. Oku, S.-I. Cho, M. Tatetsu, M. Abiko, M. Mamada, T. Sakanoue, Y. Suzuri, J. Kido and S. Tokito, "A Solution-Processed Organic Thin-Film Transistor Backplane for Flexible Multiphoton Emission Organic Light-Emitting Diode Displays”, IEEE Electron Device Letters, vol.36, no.8, (2015), pp.841-843.

[10] Y.-J. Pu, T. Chiba, K. Ideta, S. Takahashi, N. Aizawa, T. Hikichi and J. Kido, "Fabrication of Organic Light-Emitting Devices Comprising Stacked Light-Emitting Units by Solution-Based Processes", Advanced Materials, vol.27, no.8, (2015), pp.1327-1332.

[11] M. Mizukami, S.-I. Cho, K. Watanabe, M. Abiko, Y. Suzuri, S. Tokito, and J. Kido, "Flexible Organic Light-Emitting Diode Displays Driven by Inkjet Printed High Mobility Organic Thin Film Transistors", IEEE Electron Device Letters, (2017), Accepted.

[12] S.-I. Cho, S.-D. Yeo, S.-K. Kim and M. Yokoyama, "Optimization of Control Block of 3-bit PWM using Adiabatic Dynamic CMOS Logic for OLED Illumination System Based on Internet of Things Service", International Journal of Smart Home, vol.10, no.9, (2016), pp.317-328.

[13] K. Fukuda, Y. Takeda, Y. Yoshimura, R. Shiwaku, L. T. Tran, T. Sekine, M. Mizukami, D. Kumaki and S. Tokito, "Fully-printed High-performance Organic Thin-film Transistors and Circuitry on Onemicron-thick Polymer Films", Nature Communications, vol.5, (2014), 4147.

[14] T. Sekitani, M. Takamiya, Y. Noguchi, S. Nakano, Y. Kato, T. Sakurai and T. Someya, "A Large-area Wireless Power-transmission Sheet using Printed Organic Transistors and Plastic MEMS Switches", Nature Materials, vol.6, (2007), pp.413-417.

[15] A. F. Vornbrock, D. Sung, H. Kang, R. Kitsomboonloha and V. Subramaian, "Fully Gravure and Ink-jet Printed High Speed pBTTT Organic Thin Film Transistors", Organic Electronics. vol.11, no.12, (2010), pp.2037-2044.

[16] K. Fukuda, Y. Yoshimura, T. Okamoto, Y. Takeda, D. Kumaki, Y. Katayama and S. Tokito, "ReverseOffset Printing Optimized for Scalable Organic Thin-Film Transistors with Submicrometer Channel Lengths", Advanced Electronic Materials, vol.1, no.8, (2015), 1500145.

[17] N. Noda, N. Kobayashi, M. Katsuhara, A. Yumoto, S. Ushikura, R. Yasuda, N. Hirai, G. Yukawa, I. Yagi, K. Nomoto and T. Urabe, "An OTFT-driven Rollable OLED Display", Journal of the Society for Information Display, vol.19, no.4, (2011), pp.316-322.

[18] W.-J. Wu, L.-R. Zhang, Z.-P. Xu, L. Zhou, H. Tao, J.-H. Zou, M. Xu, L. Wang and J.-B. Peng, "A HighReliability Gate Driver Integrated in Flexible AMOLED Display by IZO TFTs", IEEE Transactions on Electron Devices, vol.64, no.5, (2017), pp.1991-1996.

[19] L. Dulau, S. Pontarollo, A. Boimond, J. -F. Garnier, N. Giraudo and O. Terrasse, "A New Gate Driver Integrated Circuit for IGBT Devices with Advanced Protections", IEEE Transactions on Power Electronics, vol.21, no.1, (2006), pp.38-44.

[20] K. Mizuuchi, H. Kawasato, M. Takeuchi, S. Inaba and T. Teramoto, "High-resolution Cardo Polymer Dielectric "VPA-series", Proceedings of 52nd Electronic Components and Technology Conference (ECTC), pp.924-930, (2002).

[21] Y. Takeda, Y. Yoshimura, Y. Kobayashi, D. Kumaki, K. Fukuda and S. Tokito, "Integrated Circuits using Fully Solution-processed Organic TFT Devices with Printed Silver Electrodes", Organic Electronics, vol.14, no.12, (2013), pp.3362-3370.

[22] H. E. Fargher and P. J. Mole, "The Implementation of a 3 Terminal SOSFET Model in SPICE for Circuit Simulation", GEC VLSI Research Laboratory, MOS1 Division.

[23] T. Nakamura, H. Matsuhashi and Y. Nagatomo, "Silicon on Sapphire (SOS) Device Technology", Oki Technical Review, Issue 200, vol.71, no.4, (2004), pp.66-69 . 


\section{Authors}

Seung-Il Cho, he was born in Gwang-ju, Korea, on August 9, 1980. He received his B.S. and M.S. degrees in marine electronics and communication engineering from Mokpo National Maritime University, Korea in 2006 and 2008, respectively. He was an associate research engineer in the R\&D division of Tomato LSI from 2008 to 2010. He received his Ph.D. degree in bio-systems engineering, Graduate School of Science and Engineering, from Yamagata University, Japan in 2013. He is currently an Assistant Professor in the Innovation Center for Organic Electronics, Yamagata University. His research interests include adiabatic dynamic CMOS logic (ADCL), digital/analog integrated circuit design, organic thin film transistor (OTFT) circuit design, and organic light emitting diode (OLED) flexible displays. He is a member of The Korea Institute of Electronic Communication Sciences.

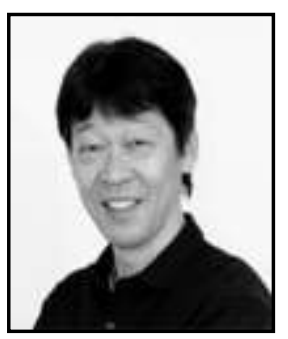

Makoto Mizukami, he was born in Nagano, Japan, on February 10, 1960. He received his B.E. degree in electronic engineering from Saitama University in 1982. From April 1982 to March 2011, he was with the development and research center at Victor Company of Japan, Limited. In June 2011, he joined Yamagata University, Yonezawa, Japan. $\mathrm{He}$ is currently an Associate Professor at the Innovation Center for Organic Electronics, Yamagata University. His research interests include organic thin film transistors (OTFTs) and organic light emitting diode (OLED) flexible displays. He is a member of The Japan Society of Applied Physics. 